\title{
Thermal unfolding of proteins
}

\author{
Marek Cieplak and Joanna I. Sułkowska \\ Institute of Physics, Polish Academy of Sciences, Al. Lotników 32/46, 02-668 Warsaw, Poland
}

\begin{abstract}
Keywords: conformational changes in proteins; Go model; molecular dynamics, titin

PACS numbers: 82.37.Rs, 87.14.Ee, 87.15.-v
\end{abstract}

\begin{abstract}
Thermal unfolding of proteins is compared to folding and mechanical stretching in a simple topology-based dynamical model. We define the unfolding time and demonstrate its low-temperature divergence. Below a characteristic temperature, contacts break at separate time scales and unfolding proceeds approximately in a way reverse to folding. Features in these scenarios agree with experiments and atomic simulations on titin.
\end{abstract}


Conformational changes in proteins occur in a variety of processes such as folding, mechanically induced stretching, chemical denaturation, and thermally induced unfolding. Standard molecular dynamics simulations of these processes cover nanosecond intervals which usually misses the relevant time scales by at least six orders of magnitude. One may gain insights into the long time scale conformational dynamics by considering effective coarse-grained models. Among these, the simplest and yet often succesful are the topology based models. They are constructed based on the knowledge of the experimentally established native conformations $[1,2]$. The topology based models offer a possibility to study various processes within one unified approach, an opportunity to explore relationships between them, and an easy way to determine dependence on parameters, such as the temperature.

In this Letter, we focus on thermal unfolding. This phenomenon is often invoked in theoretical searches for a molecular interpretation of the transition state for the folding process [3]. The transition state is probed experimentally through the protein-engineering-based so called $\phi$-value analysis [4]. The assumptions underlying the theoretical search for the transition state by simulating thermal unfolding are that unfolding should proceed in a way that is reverse to folding and that the transition state should be quickly accessible from the native state, especially if high temperatures are applied (even up to $200^{\circ} \mathrm{C}$ which in itself may, however, alter the free energy landscape of a protein significantly). Additional assumptions attempt to relate the transition state to "large structural changes" $[5,6]$ in an unfolding evolution of a protein - a point recently assessed in Ref. [7]. Here, we analyse thermal unfolding within the topology-based model as implemented in Refs. [8] and [9]. We characterize unfolding at various temperatures by determining unfolding times and by providing scenarios of unfolding. We show that there is a characteristic temperature, $T_{\Omega}$, associated with unfolding above which rupturing of bonds occurs simultaneously (on an average) at all sequential separations. We show that the unfolding times diverge on lowering the temperature and that below $T_{\Omega}$, the unfolding process runs in reverse to folding as mon- 
itored at an optimal folding temperature. Some of our predictions regarding scenarious of the conformational changes are found to be consistent with experimental findings.

We consider several model proteins with a special emphasis on the I27 globular domain of the muscle protein titin (the Protein Data Bank [10] code 1tit). Mechanical stretching of this protein has been extensively studied in experiments involving atomic force microscopy [11-13] and there is also some information about its folding [14,15]. Furthermore, we have already studied it undergoing both processes through molecular dynamics simulations within the topology based model $[9,16-18]$.

This model can be outlined as follows. The protein is represented by the $\mathrm{C}^{\alpha}$ atoms that are tethered by harmonic potentials with minima at $3.8 \AA$. The native contacts are described by the Lennard-Jones potentials $V_{i j}=4 \epsilon\left[\left(\frac{\sigma_{i j}}{r_{i j}}\right)^{12}-\left(\frac{\sigma_{i j}}{r_{i j}}\right)^{6}\right]$, where the length parameters $\sigma_{i j}$ are chosen so that the potential minima correspond, pair-by-pair, to the native distances between the $\mathrm{C}^{\alpha}$ atoms $i$ and $j$. Which amino acids form native contacts is determined through atomic overlaps as described by Tsai et al. [19]. The non-native contacts are described by repulsive cores of $\sigma=5 \AA$. The energy parameter, $\epsilon$, is taken to be uniform and its effective value appears to be of order $900 \mathrm{~K}$, at least for titin. The optimal folding temperature, $T_{\min }$, for I27 has been found to correspond to the reduced temperature $\tilde{T}=k_{B} T / \epsilon$ of 0.275 [9] ( $k_{B}$ is the Boltzmann constant and $T$ is temperature) which is close to the room temperature value of $\tilde{T}=0.3$. In our stretching simulations, both ends of the protein are attached to harmonic springs of elastic constant $k=0.12 \epsilon / \AA^{2}$ which is close to the values corresponding to the elasticity of experimental cantilevers. The free end of one of the two springs is constrained while the free end of the second spring is pulled at constant speed, $v_{p}$, along the initial end-to-end position vector. We focus on $v_{p}$ of $0.005 \AA / \tau$, where $\tau=\sqrt{m \sigma^{2} / \epsilon} \approx 3 \mathrm{ps}$ is the characteristic time for the Lennard-Jones potentials. Here, $\sigma=5 \AA$ is a typical value of $\sigma_{i j}$ and $m$ is the average mass of the amino acids. Thermostating is provided by the Langevin noise which also mimics random kicks by the implicit solvent. An equation of 
motion for each $\mathrm{C}^{\alpha}$ reads $m \ddot{\mathbf{r}}=-\gamma \dot{\mathbf{r}}+F_{c}+\Gamma$, where $F_{c}$ is the net force on an atom due to the molecular potentials. The damping constant $\gamma$ is taken to be equal to $2 \mathrm{~m} / \tau$ and the dispersion of the random forces is equal to $\sqrt{2 \gamma k_{B} T}$. This choice of $\gamma$ corresponds to a situation in which the inertial effects are negligible [8] but the damping action is not yet as strong as in water. Increasing $\gamma$ twentyfold results in a twentyfold increase in the time scales bringing the typical value of $v_{p}$ within two orders of magnitude of the experimental pulling speeds [9] and correspondingly longer folding times [8]. The equations of motion are solved by a fifth order predictor-corrector scheme.

The top two panels of Figure 1 illustrate what happens to distances between two pairs of amino acids that make native contacts when submitting the I27 domain of titin to a very high reduced temperature of 1.1 . The broken line corresponds to the distance of $1.5 \sigma_{i j}$ that is considered as a qualitative treshold for the amino acids staying or not staying in a contact $[8,16]$. It is seen that the treshold line is being crossed repeatedly due to thermal fluctuations. However, there is a well defined and pair-specific time $t_{u}$ at which the contact breaks for good, at least within the unfolding time, $t_{\omega}$ that is defined by the requirement that all non-local contacts are broken. Specifically, the non-locality refers to the sequencial distance $|j-i|>l$, where $l=4$ (non-helical). The example values of $t_{\omega}$ are indicated in the top panels of Figure 1. The unfolding scenarios may be defined in terms of a list of the times $t_{u}$ that are averaged over several hundred different trajectories.

The values of $t_{\omega}$ vary across the trajectories and we define $t_{\Omega}$ as their median (a simple average would be ill-defined if there was no unfolding within a cutoff duration in the time evolution). The lower left panel of Figure 1 shows the temperature dependence of $t_{\Omega}$ for three model proteins including the I27 domain of titin. On lowering the $\tilde{T}, t_{\Omega}$ grows rapidly, faster than according to the Arrhenius law, suggesting perhaps a Vogel-Fulcher-like divergence at a finite $\tilde{T}_{0}$. However, a finite system such as a single protein can give rise to a divergence only at $\tilde{T}=0$. This statement also applies to folding times which generally 
have a U-shaped temperature dependence with divergences at zero and infinity with the former being Arrhenius-like [20,8] (mean field theories may lead to different conclusions). The $\tilde{T}$-dependence of $t_{\Omega}$ appears to be consistent, to a leading order with the $\exp \left(A / T^{2}\right)$ law. Interpretation of this law, and corrections to it, remain to be elucidated. Our data do not rule out a power law divergence either.

We have found that varying the parameter $l$ between 4 and 10 affects $t_{\Omega}$ insignificantly. However reducing $l$ below 4 results in a substantially different $t_{\Omega}$ as shown in the lower right panel of Figure 1 for the 1 bba protein for $l=2$. This suggests a physical relevance of $l=4$ for distinguishing between local and non-local contacts [21]. Considering $l$ smaller than 4 is impractical computationally for proteins that are bigger than 1bba, and appears to have no justification in the chemical denaturation [22]. The lower right panel of Figure 1 shows that the divergence of the unfolding time also applies to secondary structures. In that theoretical case, $l=2$ is a more sensible choice to take.

There are characteristic temperatures that are associated with the processes of folding and stretching. In the case of folding, it is the temperature of kinetic optimality $(0.275$ for 1tit). In the case of stretching, it is the temperature (0.8 for 1tit) at which the purely entropic behavior [23] sets in: above it, force peaks disappear and the system acquires the worm-like-chain behavior [24]. Is there a characteristic temperature, $T_{\Omega}$ that can be associated with thermal unfolding? Figure 2 suggests that there indeed such a temperature exists and its reduced value for titin is around 1.1. Below this temperature, the median and the most probable unfolding times diverge from each other significantly, indicating emergence of a broad distribution of time scales and temporal separation of the unfolding events.

The left top panel of Figure 3 shows the average scenarios of the unfolding events in titin at two temperatures: at $T_{\Omega}$ and substantially below it, i.e., at $\tilde{T}=0.85$. These scenarios show the average unfolding times, $t_{U}=<t_{u}>$ of specific contacts. These times are plotted 
against the contact order, i.e., against the sequential distance $|j-i|$. In order to see the details in the scenarios, we actually plot $t_{U}-t_{\Omega}$ which removes the dominant time scale. We observe that at and above $T_{\Omega}$, the thermal fluctuations destroy bonds nearly simultaneously, independent of the contact order. On the other hand, below $T_{\Omega}$, the scenarios acquire reproducible structures in which the contacts between strands $\mathrm{C}$ and $\mathrm{F}$ (solid squares) and between strands A and G (solid circles) disintegrate much sooner, on an average, than those between strands B-E (open triangles) and B-G (open circles). This order of events agrees with all-atom nanosecond long unfolding simulations of titin by Paci and Karplus [25] in which several trajectories were studied at $450 \mathrm{~K}$.

In broad features, the unfolding scenario at $\tilde{T}=0.85$ runs in reverse to the folding scenario at $T_{\min }$ shown in the top right panel of Figure 3 . However, the cross correlation plot between the two scenarios, shown in the bottom left panel of Figure 3, indicates that the two processes are not simply unticorrelated but merely reflecting the time flow of events in a fairly monotonic fashion. It is interesting to note that the events which are most relevant to the search of the transition state - the final stages of folding and the initial stages of unfolding - anticorrelate in a nearly linear way. This point qualitatively agrees with allatom simulations for the $\beta$-hairpin fragment of protein $\mathrm{G}$ [3] that were performed between certain characteristic sets of conformations (16 amino acids were considered, the unfolding simulations took place at $350 \mathrm{~K})$.

The folding scenario shown in the top right panel of Figure 3 is defined in terms of average times, $t_{c}$, at which specific contacts are established for the first time [8]. (Folding is considered to be fully accomplished when all contacts are simultaneously established for the first time). We have found that folding in the model titin takes place in two channels. In the first channel, comprising about $24 \%$ of the trajectories, the C-F contacts are established in twice as long a time as needed to set the A-G and A'-G contacts. In the second channel, comprising the remaining $76 \%$ of the trajectories (at the temperature of optimal folding), 
C-F gets established somewhat earlier than the A-G contacts. The scenario shown in Figure 11 of Reference [9] combines the two channels. The scenario shown here discards the minority channel. Our studies of a generalized model of titin, in which the $\mathrm{C}^{\beta}$ atoms are also included in the description of the model, agrees qualitatively with the majority-channel scenario. This updated scenario is consistent with the $\phi$-value data $[14,15]$.

Finaly, we consider mechanical stretching of 1 tit at constant speed. Its scenario is defined in terms of the last average distance, $d_{u}$, at which contacts are still holding when the C-terminus is moving at a constant speed and the N-terminus is attached to an elastic anchor. We have already established that stretching at "room temperature" proceeds in a way that is unrelated to folding $[16,9]$. It is only in the entropic limit, when stretching is governed exclusively by the sequential distance that stretching is approximately reverse to folding at optimality [23]. The right bottom panel of Figure 4 shows that when unfolding and stretching are both done at $\tilde{T}=0.85$ they follow each other in a monotonic way. The order of events is nearly identical but the time intervals between them do not scale linearly except perhaps at the very begining of the two processes. The reduced temperature of 0.85 belongs to the entropic regime but, at the same time, it is below $T_{\Omega}$. The inset of this panel shows, however, that stretching at at the "room temperature" value of $\tilde{T}=0.3$ does not correlate with unfolding at $\tilde{T}=0.85$ at all.

In summary, we have provided an operational definition of the unfolding times, demonstrated their "low"-temperature (faster than Arrhenius) divergence and indicated existence of a characteristic temperature below which unfolding scenarios have contact-order-related structure and time scales become broadly distributed. We have demonstrated that long time folding events are anticorrelated with the short time unfolding events. We find that the simple topology-based dynamical models qualitatively capture what is known from experiments and simulations about the average order in which conformational changes proceed in titin. 


\section{ACKNOWLEDGMENTS}

We appreciate discussions with Jane Clarke and Piotr Szymczak. This work was funded by the Ministry of Science in Poland (grant 2P03B 03225). 


\section{REFERENCES}

[1] H. Abe and N. Go, Biopolymers 201013 (1981).

[2] S. Takada, Proc. Natl. Acad. Sci. (USA) 9611698 (1999).

[3] see, e.g. V. S. Pande and D. S. Rokhsar, Proc. Natl. Acad. Sci. (USA) 96: 9062-9067 (1999).

[4] A. R. Fersht, Structure and Mechanism in Protein Science: A Guide to Enzyme Catalysis and Protein Folding, New York, Freeman (1998).

[5] A. Li, and V. Daggett, Proc. Natl. Acad. Sci. (USA) 91: 10430-10434 (1994).

[6] S. L. Kazmirski, K.-B. Wong, S. M. V. Freund, Y.-J. Tan, A. R. Fersht, and V. Daggett, Proc. Natl. Acad. Sci. (USA) 98: 4349-4354 (2001).

[7] I. Chang, M. Cieplak, J. R. Banavar, and A. Maritan Protein Science 13 2446-2457 (2004).

[8] M. Cieplak and T. X. Hoang, Biophysical J. 84475 (2003).

[9] M. Cieplak, T. X. Hoang and M. O. Robbins, Proteins: Struct. Funct. Bio. 56285 (2004).

[10] F. C. Bernstein, T. F. Koetzle, G. J. B. Williams, E. F. Meyer Jr., M. D. Brice, J. R. Rodgers, O. Kennard, T. Shimanouchi, and M. Tasumi M, J. Mol. Biol. 112535 (1997).

[11] M. Rief, M. Gautel, F. Oesterhelt, J. M. Fernandez JM, and H. E. Gaub, Science 276 1109 (1997).

[12] P. E. Marszalek, H. Lu, H. B. Li, M. Carrion-Vazquez, A. F. Oberhauser, K. Schulten, and J. M. Fernandez, Nature 402100 (1999).

[13] S. B. Fowler, R. B. Best, J. L. Toca Herrera, T. J. Rutherford, A. Steward, E. Paci, M. 
Karplus M, and J. Clarke, J. Mol. Biol. 322, 841 (2002).

[14] S. B. Fowler, and J. Clarke, Structure 9, 355 (2001).

[15] C. D. Geierhaas, E. Paci, M. Vendruscolo, and J. Clarke, J. Mol. Biol. 343 1111-1123 (2004).

[16] M. Cieplak, T. X. Hoang and M. O. Robbins, Proteins: Struct. Funct. Bio. 49114 (2002).

[17] M. Cieplak, A. Pastore and T. X. Hoang, J. Chem. Phys. 122054906 (2004).

[18] M. Cieplak, Physica A 35228 (2004).

[19] J. Tsai, R. Taylor, C. Chothia, and M. Gerstein, J. Mol. Biol. 290253 (1999).

[20] N. D. Socci and J. N. Onuchic, J. Chem. Phys. 101 1519-1528 (1994).

[21] The relevance of this value of $l$ also emerges in the context of the tube model of proteins, see J. R. Banavar, M. Cieplak, and A. Maritan, Phys. Rev. Lett. 93238101 (2004).

[22] D. Shortle and M. S. Ackerman, Science 293 487-489 (2001).

[23] M. Cieplak, T. X. Hoang and M. O. Robbins, Phys. Rev. E 69011912 (2004).

[24] M. Doi and S. F. Edwards. 1988, Theory of polymer dynamics, Oxford University Press, Oxford.

[25] E. Paci and M. Karplus, Proc. Natl. Acad. Sci. (USA) 97 6521-6526 (2000). 


\section{FIGURE CAPTIONS}

Fig. 1. The top panels show examples of evolution of the distance, $d_{i j}$, between two contact making amino acids $i$ and $j$ in the I27 (1tit) domain of titin when starting from the native structure and then applying a temperature of $\tilde{T}=1.1$. The contacts involved are between the $\beta$-strands $\mathrm{C}, \mathrm{F}$ and $\mathrm{B}, \mathrm{G}$. The A, A', B, C, D, E, F, and G strands in titin correspond to the sequential segments 4-7, 11-15, 18-25, 32-36, 47-52, 55-61, 69-75, and 78-88 respectively. This protein consists of altogether 89 amino acids. The lower panels show $t_{\Omega}$ for the systems indicated. The lower left (right) panel refers to calculations done with the $l=4(l=2)$ criterion. The data are generally based on at least 201 trajectories; above $\tilde{T}$ of 0.8 - on at least 501 trajectories. There are two data sets for titin. The solid symbols correspond to the Go-like model discussed in this paper whereas the open symbols correspond to a generalized Go-like model with side groups in which the degrees of freedom related to the $\mathrm{C}^{\beta}$ atoms are included. The generalized model shows a similar behavior. The lines in the lower panel illustrate fits to the $t_{\Omega} / \tau=\exp \left(A / \tilde{T}^{2}-B / \tilde{T}+C\right)$ law, where the sets $(A, B, C)$ are $(10.592,10.492,6.976)$ for 1 tit, $(8.381,9.887,6.258)$ for $1 \mathrm{crn},(1.391,0.943,1.548)$ for $1 \mathrm{bba}$ with $l=4,(5.259,1.576,4.916)$ for 1 bba with $l=2,(2.329,0.671,0.659)$ for the hairpin, and $(3.363,2.143,2.585)$ for the helix. The fitting confidence level is at least 0.987 . Somewhat poorer fits were obtained by using the $\exp (\exp (D / \tilde{T})$ law. The data points for the $C^{\alpha}$-based model can also be superficially fitted to the Vogel-Fulcher-like divergences with the apparent $\tilde{T}_{0}$ of $0.56,0.44$, and 0.20 and with the energy barriers of 2.6 2.3 and 1.5 for 1 tit, $1 \mathrm{crn}$, and 1 bba respectively.

Fig. 2. The distribution of unfolding times for 1 tit for the three temperatures indicated. The arrows point at the median values. The inset in the middle panel shows the temperature dependence of the difference between the median and peak values in the distributions. 
Fig. 3. The top left and right panels show the unfolding and folding scenarios in 1 tit respectively. The data are averaged over 501 trajectories. The unfolding scenarios are shown for the two temperatures indicated. The broken line separates the data points obtained for the two temperatures. The folding scenario corresponds to the temperature of the fastest folding. The symbols assigned to specific contacts are the same in both panels. Open circles, open triangles, open pentagons, solid circles, and solid squares correspond to contacts B-G, B-E, D-E, A-G, and C-F respectively. The stars denote all other contacts. The lower left panel cross-plots the folding scenario with the unfolding scenario at the temperatures indicated. The lower right panel crossplots events of mechanical stretching with those of thermal unfolding. The mechanical stretching in the inset and the main panel is performed at $\tilde{T}=0.3$ and 0.85 respectively. 


\section{FIGURES}
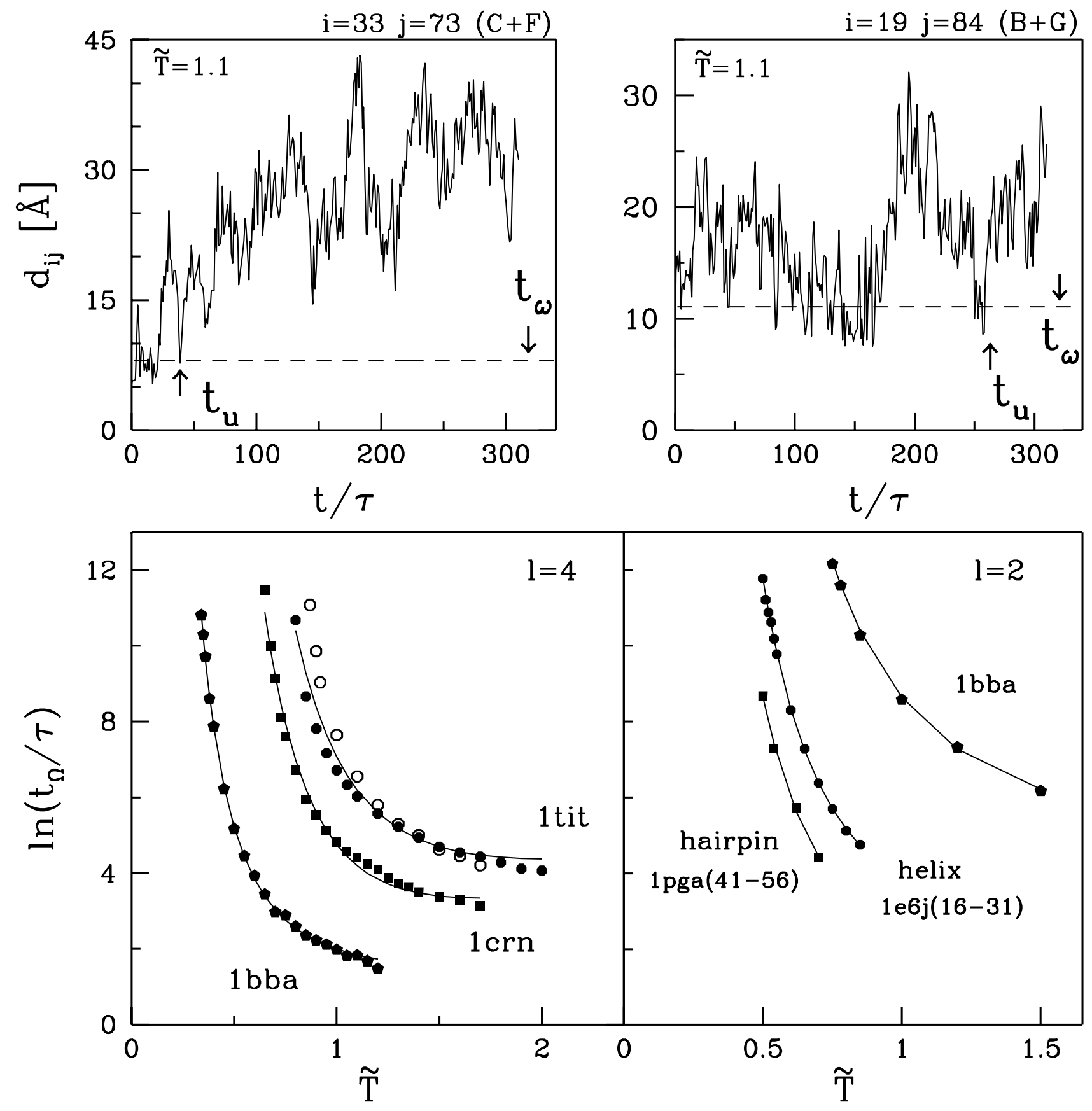

FIG. 1. 


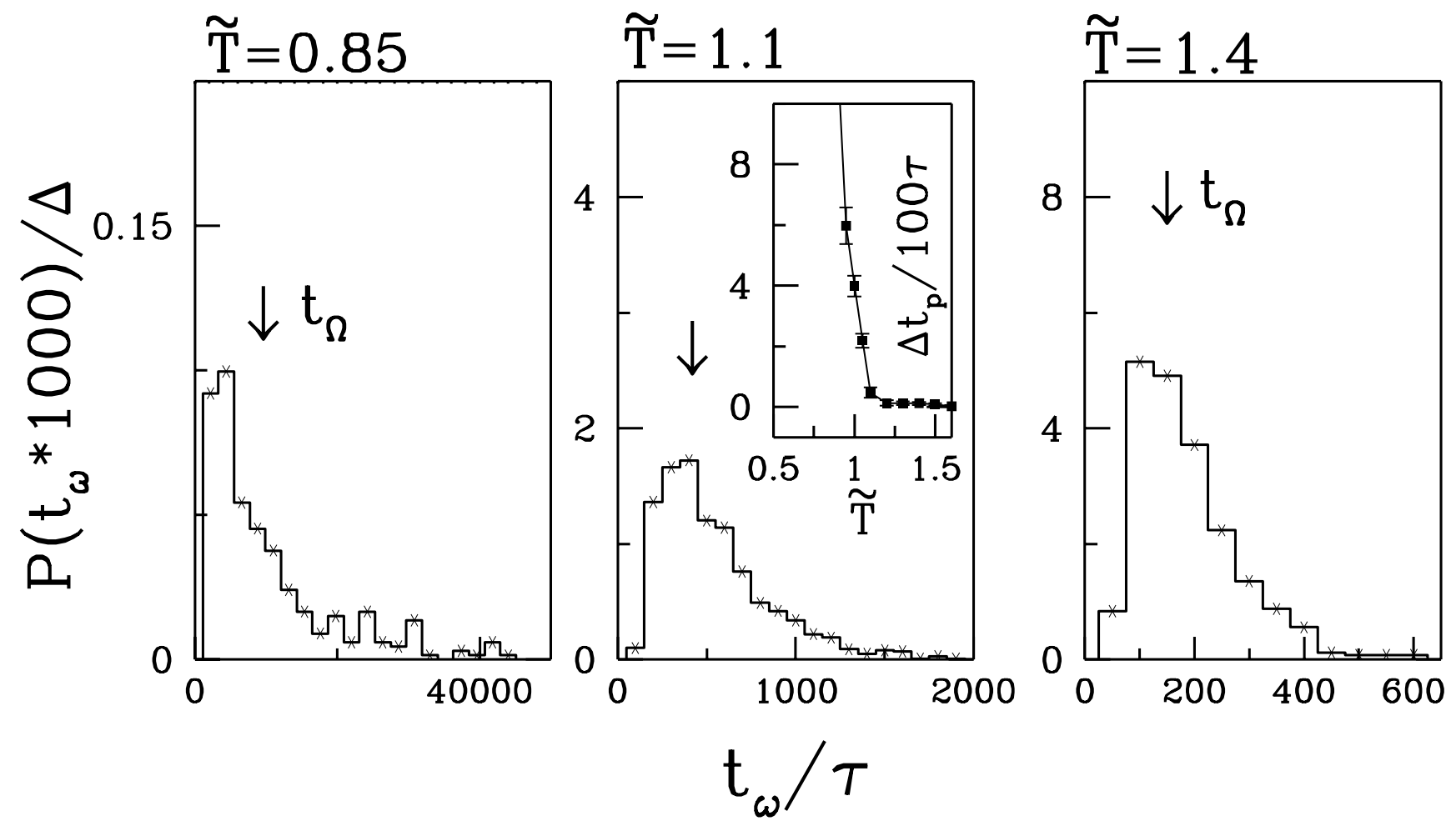

FIG. 2. 

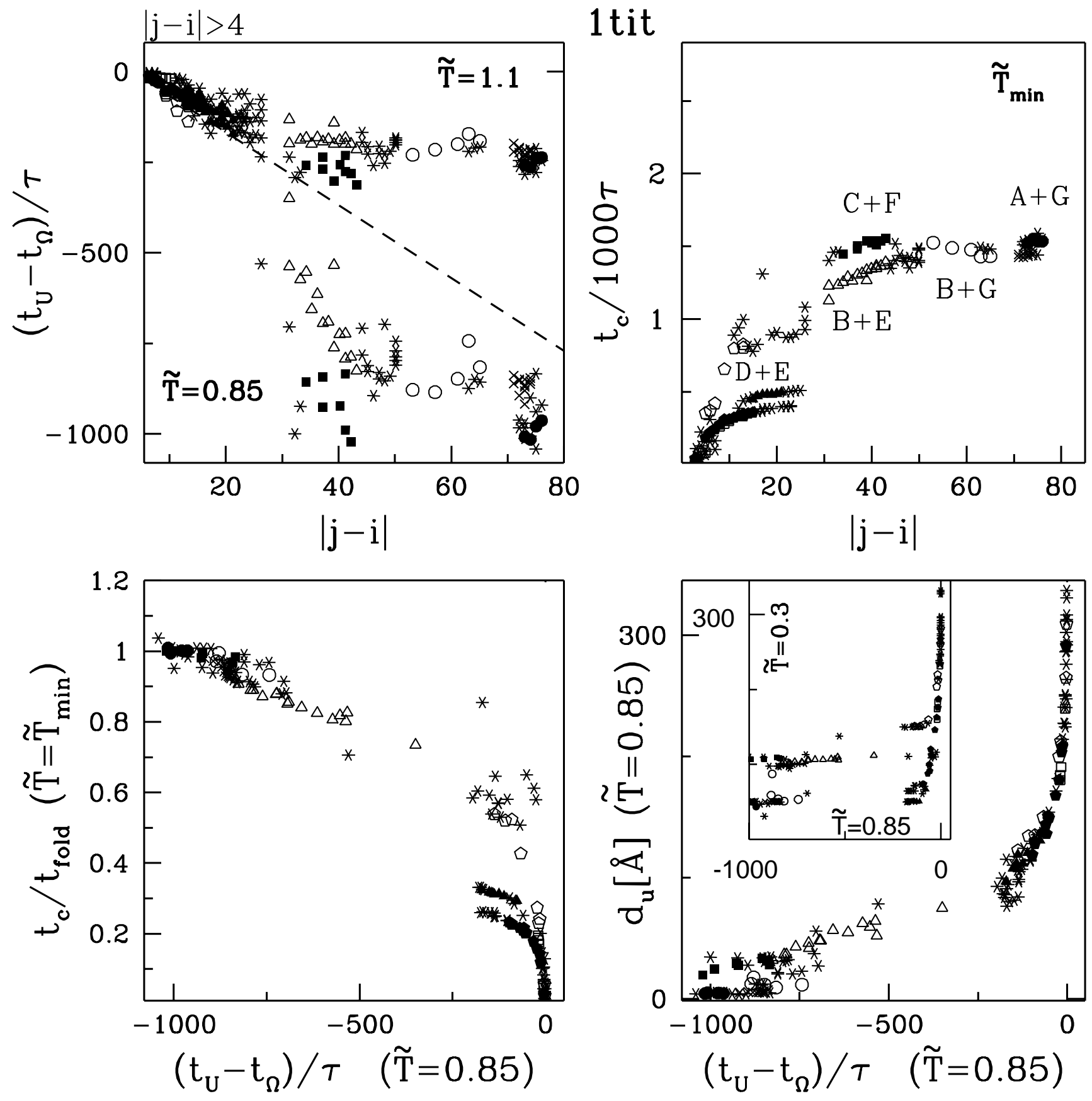

FIG. 3. 